\title{
Diagnostic Accuracy of Ultrasound for the Evaluation of Ureteric Calculi Taking Non-enhanced Computed Tomography as a Gold Standard
}

\author{
Aqsa Rao* \\ Bachelors in Medical Imaging Technology, Student, Department of Allied Health Sciences, Superior College
} Lahore, University Campus, 17-KM Raiwaind Road, Kot Arain, Lahore, Pakistan

Afeera Toor

Bachelors in Medical Imaging Technology, Student, Department of Allied Health Sciences, Superior College Lahore, University Campus, 17-KM Raiwaind Road, Kot Arain, Lahore, Pakistan

Marriam Fatima

Bachelors in Medical Imaging Technology, Student, Department of Allied Health Sciences, Superior College Lahore, University Campus, 17-KM Raiwaind Road, Kot Arain, Lahore, Pakistan

Jasia Noor

Bachelors in Medical Imaging Technology, Student, Department of Allied Health Sciences, Superior College Lahore, University Campus, 17-KM Raiwaind Road, Kot Arain, Lahore, Pakistan

Ramisha Sabir

Bachelors in Medical Imaging Technology, Student, Department of Allied Health Sciences, Superior College Lahore, University Campus, 17-KM Raiwaind Road, Kot Arain, Lahore, Pakistan

Sybil Rose

Masters in Diagnostic Ultrasound, Lecturer, Department of Allied Health Sciences, Superior College Lahore, University Campus, 17-KM Raiwaind Road, Kot Arain, Lahore, Pakistan

Muhammad Fiaz Ahmed

Head of Department, Ali Fatima Hospital, Lahore, Pakistan

Rana Muhammad Athar Azeem Shams

Masters in Medical Imaging Technology, Lecturer, Department of Allied Health Sciences, Superior College Lahore, University Campus, 17-KM Raiwaind Road, Kot Arain, Lahore, Pakistan

Rana Muhammad Bakhtawar Khan Sajawal

Masters in Mathematics, Lecturer, Department of Allied Health Sciences, Superior College Lahore, University Campus, 17-KM Raiwaind Road, Kot Arain, Lahore, Pakistan

Ambreen Sadaf

Masters in Clinical Psychology, Lecturer, The University of Lahore, 1-KM Defence Road, near Bhuptian Chowk, Lahore, Pakistan

The research is financed by Asian Development Bank. No. 2006-A171(Sponsoring information)

\section{Abstract}

Objective: To find the diagnostic accuracy of ultrasound for the evaluation of ureteric calculi taking non enhanced Computed Tomography as gold standard.

Study Design: An analytical cross-sectional prospective study was performed.

Settings: The study was performed in National Hospital and Medical Center, Lahore.

Period: An analytical cross-sectional study was conducted from 15 October, 2020 to 15 January, 2021.

Material \& Methods: In our study, all those patients with suspected ureteric calculi presenting to radiology department were included. Patients on dialysis, those with polycystic disease and ureteric stricture, as well as patients who declined to give consent and those who were uncooperative, were all excluded. Ultrasound was performed on both sides of the patient's kidney, concentrating on the ureters. Then were sent to a 64 slice CT scanner for a non-enhanced CT scan.

Results: Total 121 Patients were reviewed in this research. Females were $82(67.8 \%)$ while male was 39 (32.2\%). On Ultrasound 61(50.4\%) were positive for ureteric calculi while $60(49.6 \%)$ were negative for stone in the ureter. 
On CT scan $110(90.9 \%)$ patients were positive for ureteric calculi while $11(9.1 \%)$ were negative for stone in ureter $(\mathrm{p}=0.05)$. The trans-abdominal ultrasound with sensitivity $58.62 \%$, Specificity $56.76 \%$, Positive predictive value $51.52 \%$ and Negative predictive value of $63.64 \%$ and accuracy of $57.68 \%$.

Conclusion: The sensitivity of ultrasound is comparable to Computed Tomography findings, hence, it can be used as an option in cases where CT scans are not available or is contraindicated, such as in pregnant women. Moreover, Ultrasound modality is cheap, with no radiation dose and is readily available in our setups, making it suitable for initial diagnosis as well as for follow-up scans.

Keywords: Non-enhanced Computed Tomography, Ultrasound, Ureteric Calculi, radiation, Computed Tomography.

DOI: $10.7176 / \mathrm{JHMN} / 90-04$

Publication date:June $30^{\text {th }} 2021$

\section{Introduction}

Ureteric calculi are a medical condition that affects the urinary tract which is resulted from solid concentrations produced by an interference in balance between solubility and precipitation of salts in urinary tract ${ }^{(1)}$. Urolithiasis is defined as the detection of stone formation in urinary tract that is detected during imaging studies like in ultrasound, CT with or without contrast or IVU ${ }^{(2)}$. Its prevalence is relatively high in America which accounts for $12 \%$ in men and $7 \%$ in women, the risk is more with the previous history of ureteric stones and is there is a family history ${ }^{(3)}$. More commonly seen in patients within age group of 35-45. American guideline recommends CT scan as first choice for ureteric stone diagnosis while European says the ultrasound as the initial modality ${ }^{(4)}$. Normal renal function is critical because it involves the filtration and excretion of metabolic waste products such as urea and ammonium; electrolyte regulation; fluid and acid-base balance; aids in the formation of red blood cells, which is used to maintain blood pressure through the renin-angiotensin-aldosterone system and to regulate water resorption; and maintain the blood pressure via the renin-angiotensin-aldosterone system and maintain the volume in blood vessels ${ }^{(5)}$. Men are more commonly affect than women. Ureteric calculi are the sixth most common condition requiring surgery in Pakistan ${ }^{(6)}$. It is estimated that up to $6 \%$ of women will experience one or more renal calculi episodes in their lives with a recurrence rate of $50 \%$. Ureteric calculi is common in patients who present with hematuria, or dysuria and acute pain located in the flank area and renal colic ${ }^{(7)}$. Non-contrast CT of low dose can predict the size and site of the calculus more accurately with the diagnosis other causes of pain of abdominal region like appendicitis, intestinal obstruction or any other things related to kidneys.

Sharad kondekar, et al in 2020, conducted a study of 150 patients were examined on ultrasound and CT and compared for the existence of calculi. Sensitivity, specificity, positive and negative predictive values of ultrasound were compared with CT as Gold standard. The size of the renal stones revealed on ultrasound was $6.8 \mathrm{~mm} \pm 3.8 \mathrm{~mm}$ and the size of stones on the CT was $3.5 \mathrm{~mm} \pm 2.7 \mathrm{~mm}$. The sensitivity and specificity of ureteric calculi on Ultrasound were $12 \%$ and $97 \%$ respectively. The specificity and sensitivity of urinary bladder calculi were 20 percent and 100 percent on USG Ultrasound is one of the best suitable and beneficial assessment tools it is simply accessible, radiation-free, reproducible, economical and non-invasive ${ }^{(1)}$. Altaf Nasir, et al in 2019, examined in 78 patients with the symptoms of ureteric calculi were seen in ultrasound and computed tomography, among the total of the patients, ureteric stones were found in $25(34.2 \%)$ patients in ultrasound. Ureteric stones were found in $52(71.2 \%)$ in computed tomography scan. The individuals of 17-75 years were mainly involved while most of the patients are male. We observed that, computed tomography scanning is better to diagnose ureteric calculi as compared to ultrasonography for ureteric calculi ${ }^{(7)}$. Fisal Ahmed et al in 2018, a total of 184 patients were suspected. Computed tomography was used to compare the specificity and sensitivity, as well as the stone size measured by US ${ }^{(8)}$.

The aim of our study was to rule-out the diagnostic efficacy of ultrasonography in detecting ureteric stones while taking Computed Tomography as a Gold standard modality for the reason that is radiation-free and it is easily available in our setups which enable the medical staff in case of emergency to relief the patient pain and start further suitable procedures, it also helps the patient who avoid radiation such as pediatric age and pregnant woman. Ultrasonography is a choice of modality for the evaluation of ureteric calculi while Computed Tomography remains as a Gold standard modality.

\subsection{Materials and Methods}

An analytical cross-sectional prospective study was performed. The study was carried out in National Hospital and Medical Center, Lahore. This duration of the study was conducted from 15 October, 2020 to 15 January, 2021. A Total of 121 patients were studied out of which 82 were females and 39 were male. Non-Probability Convenient Sampling Technique was used. The rules and regulations set by the ethical committee of The Superior College, Lahore were followed while conducting the research and the rights of the research participants were respected. Written informed consent attached was taken from all the participants. Patients with Flank Pain, Hematuria, Abdominal Pain, Polyuria, Pregnant Patient, patients in Urology Department were included in this study. Patients 
with undergoing dialysis, uncooperative patient, refuse to give consent, polycystic disease and patients with ureteric stricture were excluded from the study. In this study informed consent was obtained from each patient. We reviewed our data-base of patients who underwent KUB non-enhanced CT and KUB ultrasound. Symptoms for imaging included flank pain, increased frequency of urination, oliguria, dribbling of urine, hematuria and a history of urinary stones. Non-Enhanced CT (Toshiba 64) was performed from the abdomen to pelvis for a detailed KUB view and images reconstructed at $1 \mathrm{~mm}$ for reporting and $5 \mathrm{~mm}$ for filming. Stone size was well defined using long axis of NECT and Ultrasound. Ultrasonography was performed, through the Trans abdominal approach for all the patients with full urinary bladder. The kidneys were visualized in both sagittal and coronal planes. All findings of non-enhanced CT were considered by the advice of consultant radiologist. Data was represented with means and standard deviation of frequency and percentage where appropriate. Chi-square test was used for the comparison of qualitative data of Computed Tomography and Ultrasound. Data was entered into SPSS 25. P-value of $<0.05$ was considered significant.

Specificity, sensitivity PPV, NPV, and accuracy was calculated as

Senstivity: $\mathrm{a} /(\mathrm{a}+\mathrm{c}) \times 100$ or $\mathrm{TP} /(\mathrm{TP}+\mathrm{FN}) \times 100$

Specificity: $d /(d+b) \times 100$ or $\mathrm{TN} /(\mathrm{TN}+\mathrm{FP}) \times 100$

Positive predictive value: $\mathrm{a} / \mathrm{a}+\mathrm{b} \times 100$

Negative predictive value: $\mathrm{d} / \mathrm{c}+\mathrm{d} \times 100$

Accuracy: $a+d / a+b+c+d \times 100$

\section{Results}

Total 121 Patients were reviewed in this research. Females were $82(67.8 \%)$ while male was $39(32.2 \%)$. On Ultrasound 61(50.4\%) were positive for ureteric calculi while $60(49.6 \%)$ were negative for stone in the ureter. (Table-) On CT scan 110 (90.9\%) patients were positive for ureteric calculi while $11(9.1 \%)$ were negative for stone in ureter $(\mathrm{p}=0.05)$. The Trans abdominal ultrasound was having sensitivity $58.62 \%$, Specificity $56.76 \%$, Positive predictive value $51.52 \%$ and Negative predictive value $63.64 \%$ and accuracy $57.68 \%$.

\section{Discussion}

Ureteric calculi are often diagnosed in emergency rooms in patients who complain of flank pain, hematuria, difficulty urinating, or a burning sensation while urinating. According to American guidelines, non-enhanced CT is the gold standard modality for diagnosing Ureteric calculi, while Ultrasound is the first option modality for evaluating Ureteric calculi according to European guidelines ${ }^{(9,10)}$.

According to our study, 121 patients were examined in total. Females accounted for $82(67.8 \%)$ of the 121 participants, while males accounted for 39. (32.2 percent). Ultrasound revealed that $61(50.4 \%)$ of the patients had ureteric calculi, while 60 (49.6\%) had no ureteric calculi. On CT scan, $110(90.9 \%)$ of the patients had ureteric calculi, while $11(9.1 \%)$ of the patients had no ureteric calculi (p0.05). The sensitivity of the transabdominal ultrasound was 58.62 percent, the specificity was 56.76 percent, the PPV was 51.52 percent, the NPV was 63.64 percent, and the diagnostic accuracy was 57.68 percent.

Mehboobul Wahab et al. conducted a similar study with 80 patients and found that ultrasound sensitivity was 69.64 percent and specificity was 66.6 percent ${ }^{(6)}$. The findings of our study agree with those of Maryam et al. According to Maryam et al, the sensitivity was 65.27 percent, the specificity was 72.09 percent, the PPV was 79.66 percent, the NPV was 55.36 percent, and the diagnostic accuracy was 67.83 percent, ${ }^{(1)}$ all of which are similar to our findings.

In our opinion, abdominal ultrasonography has numerous advantages as a diagnostic tool for the detection of ureteric calculi, especially in pregnant women and infants, as well as for follow-up scans. New techniques and equipment, as well as sonographer experience, can provide additional information and help to improve ultrasonography diagnostic efficacy for ureteric calculi detection even further. The limitation of this study is that sample size was small.

\section{Conclusion}

The sensitivity of ultrasound is comparable to Computed Tomography findings, hence, it can be used as an option in cases where CT scans are not available or is contraindicated, such as in pregnant women. Moreover, Ultrasound modality is cheap, radiation-free and is readily available in our setups, making it suitable for initial as well as for follow-up scans.

\section{Conflict of interest}

No potential conflict of interest relevant to this article was reported.

\section{References}

1. Javed M. Diagnostic Accuracy of Trans-Abdominal Ultrasonography in Urolithiasis, keeping CT KUB as Gold 
Standard. Journal of Islamabad Medical \& Dental College. 2018;7(3):204-7.

2. Huang C, Chuang C, Wong Y, Wang L, Wu C. Useful prediction of ureteral calculi visibility on abdominal radiographs based on calculi characteristics on unenhanced helical CT and CT scout radiographs. International journal of clinical practice. 2009;63(2):292-8.

3. Rodger F, Roditi G, Aboumarzouk OM. Diagnostic Accuracy of Low and Ultra-Low Dose CT for Identification of Urinary Tract Stones: A Systematic Review. Urologia Internationalis. 2018;100(4):375-85.

4. Ahmed F, Askarpour M-R, Eslahi A, Nikbakht H-A, Jafari S-H, Hassanpour A, et al. The role of ultrasonography in detecting urinary tract calculi compared to CT scan. Res Rep Urol. 2018;10:199-203.

5. Asif HS, Naeem MW, Rose S, Hussain M, Iqbal R, Irfan M, et al. Ultrasonographic Correlation of Cortical Thickness and Echogenicity Among Patients Suffering From Chronic Renal Failure. Journal of Health and Medical Sciences. 2019;2(4).

6. Wahab M, Tariq K, Ishtiaq M, Ahmad K, Aslam M, Khan S. Diagnostic accuracy of ultrasound in detecting ureteric stone keeping non-enhanced CT as gold standard. Annals of Punjab Medical College (APMC). 2019;13(3):223-5.

7. Altaf N, Kamran A, Naseem B, Iqbal M, Asif R, Farooq S, et al. Diagnostic accuracy of ultrasonography versus computed tomography for ureteric calculi among the adult patients visiting Mayo Hospital Lahore. J Health Med Sci. 2019;2:68-74.

8. Kondekar S, Minne I. Comparative Study of Ultrasound and Computerized Tomography for Nephrolithiasis Detection. Radiology. 2020;5(2):B4-B7.

9. Coursey CA, Casalino DD, Remer EM, Arellano RS, Bishoff JT, Dighe M, et al. ACR Appropriateness Criteria ${ }^{\circledR}$ acute onset flank pain-suspicion of stone disease. Ultrasound quarterly. 2012;28(3):227-33.

10. Türk C, Petř́k A, Sarica K, Seitz C, Skolarikos A, Straub M, et al. EAU guidelines on interventional treatment for urolithiasis. European urology. 2016;69(3):475-82.

Table 1. Cross Tabulation of non-enhanced CT and Ultrasound

\begin{tabular}{|c|c|c|c|c|c|}
\hline & \multicolumn{2}{|c|}{ CT } & \multirow[b]{2}{*}{ Total } \\
\hline & & & Positive & Negative & \\
\hline \multirow[t]{4}{*}{ Ultrasound } & \multirow[t]{2}{*}{ Positive } & Count & 57 & 4 & 61 \\
\hline & & \% within CT & $51.8 \%$ & $36.4 \%$ & $50.4 \%$ \\
\hline & \multirow[t]{2}{*}{ Negative } & Count & 53 & 7 & 60 \\
\hline & & \% within CT & $48.2 \%$ & $63.6 \%$ & $49.6 \%$ \\
\hline \multirow{2}{*}{\multicolumn{2}{|c|}{ Total }} & Count & 110 & 11 & 121 \\
\hline & & \% within CT & $100 \%$ & $100 \%$ & $100 \%$ \\
\hline
\end{tabular}

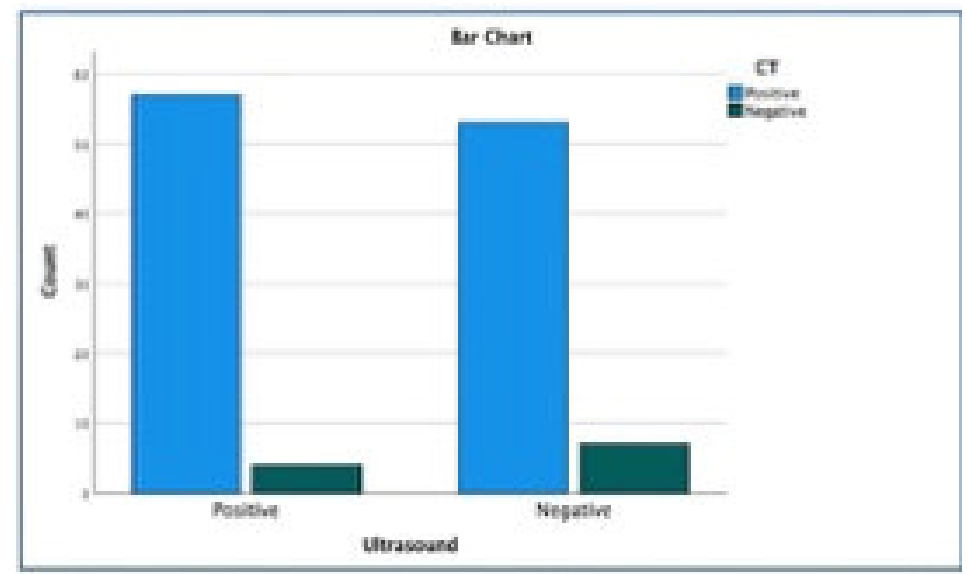

Figure 1. Frequency of CT and Ultrasound 
Table 2. Statistical values of CT and Ultrasound

\begin{tabular}{|l|l|l|}
\hline Statistic & Value & $\mathbf{9 5 \%}$ CI \\
\hline Sensitivity & $58.62 \%$ & $47.55 \%-69.08 \%$ \\
\hline Specificity & $56.76 \%$ & $47.02 \%-66.13 \%$ \\
\hline Positive likelihood Ratio & 1.36 & $1.03-1.79$ \\
\hline Negative likelihood Ratio & 0.73 & $0.54-0.98$ \\
\hline Disease Prevalence $(*)$ & $43.94 \%$ & $36.91-51.15 \%$ \\
\hline Positive Predictive value $(*)$ & $51.52 \%$ & $44.62-58.62 \%$ \\
\hline Negative Predictive value $(*)$ & $63.64 \%$ & $56.50 \%-70.22 \%$ \\
\hline Diagnostic accuracy $(*)$ & $57.58 \%$ & $50.37 \%-64.55 \%$ \\
\hline
\end{tabular}

